\title{
Performance Analysis of the Multiband Orthogonal Frequency Division Multiplexing Ultra-Wideband Systems for Multipath Fading and Multiuser Interference Channels
}

\author{
Yuan Ouyang, Wen-Piao Lin, and Chuan-Chih Liu \\ Department of Electrical Engineering, Chang Gung University, Taoyuan City 33302, Taiwan \\ Correspondence should be addressed to Wen-Piao Lin; wplin@mail.cgu.edu.tw
}

Received 25 September 2014; Revised 8 March 2015; Accepted 11 March 2015

Academic Editor: Francesco Soldovieri

Copyright (c) 2015 Yuan Ouyang et al. This is an open access article distributed under the Creative Commons Attribution License, which permits unrestricted use, distribution, and reproduction in any medium, provided the original work is properly cited.

\begin{abstract}
This paper presents the performance analysis of the multiband orthogonal frequency division multiplexing (MB-OFDM) ultrawideband (UWB) systems for multipath fading and multiuser interference channels. A closed form approximation of the BER performance of the MB-OFDM UWB system with multiple interferences is proposed. Based on the derived approximation, the effects on the BER performance for the choice of the codeword constraint lengths of the convolutional encoder, the length of the cyclic prefix, and the multiuser environments of two or more interferers are thoroughly discussed. Four UWB multipath fading channels are also investigated for the BER performance of the MB-OFDM UWB system. The simulated results provide us with useful information to appropriately choose the parameters of the MB-OFDM UWB system for the sake of achieving the BER performance that conforms to requirement of the FCC standards.
\end{abstract}

\section{Introduction}

Ultra-wideband (UWB) technology [1-4], operated in frequencies between $3.1 \mathrm{GHz}$ and $10.6 \mathrm{GHz}$, is an emerging short distance, high bandwidth, and high data rate wireless communication system. The general design approach for UWB wireless system includes the narrow time-domain pulsed, pulsed multiband, and multiple band orthogonal frequency division multiplexing (MB-OFDM) [5-7] modulation. According to previous literatures [8-11], the main disadvantage of the narrow time-domain pulsed UWB system is the high complexity of the RF circuits that capture the significant multipath energy [12-14]. The pulsed multiband UWB system also has the same difficulty in its RF chain design. However, the MB-OFDM UWB system has several advantages, including the ability to efficiently capture multipath energy, the insensitivity to group delay variations, the ability to deal with narrowband interferers, and the fewer requirements of the band-timing switching $[15,16]$. The drawback of the MB-OFDM UWB system is the high peakto-average power ratio (PAPR) of the transmitted signals. The MB-OFDM UWB systems provide the data rates from
53.3 to $480 \mathrm{Mbit} / \mathrm{s}$ by utilizing different spreading gain factors and different schemes of the forward error correction (FEC) coding and time-frequency coding (TFC). The FEC coding with a convolutional code provides the code rates of $1 / 3,11 / 32$, $1 / 2,5 / 8$, and $3 / 4$.

Multipath energy collection is the key factor of the robustness and range of the wireless communication systems. The cyclic prefix (CP) length of the OFDM system determines the received multipath energy and reduces the intercarrier interference (ICI) and intersymbol interference for multipath fading channels. However, the CP structure would lead to a serious ripple problem in the power spectral density (PSD) of the transmitted signals. The MB-OFDM UWB systems use a zero-padded prefix (ZPP) instead of the $\mathrm{CP}$ to reduce the ripple problem of the PSD and extend the transmission range of the system.

The channel models specified in IEEE 802.15.3a standard $[17,18]$ were based upon Saleh-Valenzuela (S-V) model [19], which was characterized by the cluster arrival rate, ray arrival rate within clusters, and cluster and ray decay factors. The gain coefficients of these channel models are assumed to be Rayleigh distributed. The performances of MB-OFDM 
UWB and DS-UWB systems are evaluated in [20]. A general framework for the performance analysis of MB-OFDM UWB system is provided in [21]. All of these works are based on the assumption of perfect frequency and timing synchronization. In addition, the cyclic prefix of OFDM system is assumed to be longer than the channel multipath delays. Hence, the systems would not suffer from the ISI and ICI. Since the multiuser interference would significantly affect the performance of the MB-OFDM UWB system, it is not appropriate to simply consider the multiuser interference as AWGN. In this paper, the multiuser interferences are modeled as a summation of flat Rayleigh-fading and unsynchronized OFDM signals.

In this paper, we propose an analytical model and a closed form BER performance equation of the MB-OFDM UWB systems with multiuser interferences for LOS and NLOS multipath fading channels. The effects on the BER performance for the choice of the codeword constraint lengths (CCL) of the convolutional encoder, the length of the cyclic prefix, and the multiuser environments of two or more interferers are thoroughly discussed. Four UWB multipath fading channels are also investigated for the BER performance of the MBOFDM UWB system. The simulated results give us valuable information to obtain the BER performance that conforms to requirement of the FCC standards by appropriately choosing the parameters of the MB-OFDM UWB systems.

\section{System Model and BER Analysis}

Figure 1 shows the block diagram of the MB-OFDM UWB system. In this system, the convolutional coded QPSK/QAMOFDM based modulation is combined with a multibanding approach, which divides the spectrum into several subbands. Each subband has $528 \mathrm{MHz}$ bandwidth. The transmitted OFDM symbols are time-interleaved and across the subbands by using a predefined time-frequency code. Various information data rates can be achieved by puncturing the convolutional encoder. The transmitted signal of $S(t)$ shown in Figure 1 can be expressed as [22-24]

$$
S(t)=\sqrt{\frac{E_{c}}{T}} \sum_{k=0}^{K-1} a_{k} e^{j(2 \pi / T) k t}, \quad 0<t<T,
$$

where $E_{c}$ is the energy per coded bit, $T$ is the symbol duration, $K$ is the total number of OFDM subcarriers, and $a_{k} \in$ $\{ \pm 1, \pm j\}$ is the coded data transmitted at the $k$ th subcarrier. The transmitted interference signal from the $i$ th MB-OFDM interferer can be described as

$$
u_{i}(t)=\sqrt{\frac{E_{i}}{T}} \sum_{m=0}^{K-1} a_{i m} e^{j(2 \pi / T) k t}, \quad 0<t<T,
$$

where $E_{i}$ is the energy per interference bit, $a_{i m}$ is the transmitted coded data of the $i$ th interference user at the $m$ th subcarrier, and $a_{i m} \in\{ \pm 1, \pm j\}$. All the interferences are assumed to be synchronized during the theoretical analysis. And later we will show that the performance would be almost the same when the interferences are unsynchronized.
Based on the above description, the received signal can be expressed as

$$
\begin{aligned}
r(t) & =S(t) * h(t)+\sum_{i=0}^{I-1} n_{i}(t)+W(t) \\
& =\sum_{k=0}^{K-1} S_{k}(t)+\sum_{i=0}^{I-1} u_{i}(t) * h_{i}(t)+W(t),
\end{aligned}
$$

where $i$ is the index of the MB-OFDM interference, $I$ is the total number of interferences, $S_{k}(t)$ is the received signal at the $k$ th subcarrier, and $W(t)$ denotes the complex white Gaussian noise, whose double-sided PSD is $N_{w}$ Watt/Hz. We denote by $h(t)$ the channel impulse response between the MB-OFDM transmitter and the MB-OFDM receiver and $h_{i}(t)$ the channel impulse response between the $i$ th interference and the MB-OFDM receiver. These channels are assumed to be slow fading Rayleigh channels, which means these channels could be considered constant over the symbol interval, but would vary from symbol to symbol. The multipath channels are independent of each other and the channel phase correction is assumed to be perfect. Based on Lampe's paper [8], UWB channel can be approximated as Rayleigh fading channel [11]. Hence, the channel responses could be modeled as

$$
\begin{gathered}
H(\omega)=\alpha(\omega) e^{j \theta(\omega)}, \\
H_{i}(\omega)=\alpha_{i}(\omega) e^{j \theta_{i}(\omega)}, \\
E\left\{\alpha^{2}\right\}=2 \Lambda, \\
E\left\{\alpha_{i}^{2}\right\}=2 \Lambda_{i},
\end{gathered}
$$

where the channel gains $\alpha(\omega)$ and $\alpha_{i}(\omega)$ at any given frequency $\omega$ are Rayleigh distributed and the channel phases $\theta(\omega)$ and $\theta_{i}(\omega)$ are uniformly distributed between 0 and $2 \pi$. Hence, the frequency response at the $k$ th subcarrier, $H_{k}$, is obtained as

$$
H_{k}=H\left(\frac{2 \pi}{T} k\right)=\alpha\left(\frac{2 \pi}{T} k\right) e^{j \theta((2 \pi / T) k)}=\alpha_{k} e^{j \theta_{k}},
$$

where $H_{k}$ is the channel frequency response at frequency $2 \pi k / T$. Substituting (5) into (3), we obtain the received signal at the $k$ th subcarrier

$$
\begin{aligned}
S_{k}(t) & =\sqrt{\frac{E_{c}}{T}} H_{k} a_{k} e^{j(2 \pi / T) k t} \\
& =\sqrt{\frac{E_{c}}{T}} \alpha_{k} a_{k} e^{j(2 \pi / T) k t+j \theta_{k}} .
\end{aligned}
$$

In a similar way, the channel frequency response at $m$ th subcarrier between the $i$ th interference and the MB-OFDM receiver can be represented as

$$
H_{i m}=H_{i}\left(\frac{2 \pi}{T} m\right)=\alpha_{i}\left(\frac{2 \pi}{T} m\right) e^{j \theta_{i}((2 \pi / T) m)}=\alpha_{i m} e^{j \theta_{i m}} .
$$




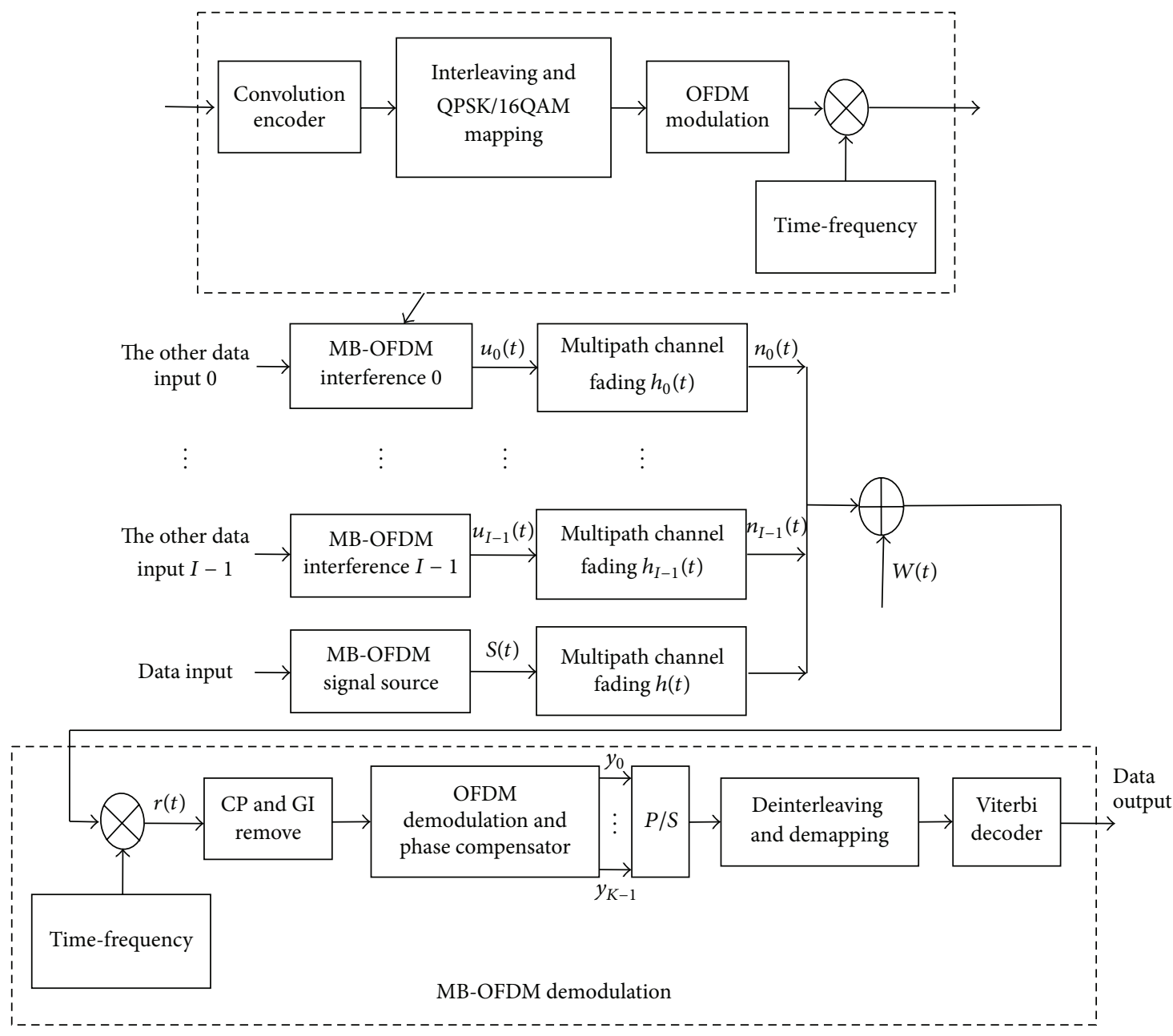

FIGURE 1: The block diagram of the MB-OFDM UWB system.

The received interference caused by the $i$ th interference, hence, can be expressed as

$$
\begin{aligned}
n_{i}(t) & =h_{i}(t) * u_{i}(t) \\
& =\sqrt{\frac{E_{i}}{T}} \sum_{m=0}^{K-1} H_{i}\left(\frac{2 \pi}{T} m\right) a_{i m} e^{j(2 \pi / T) m t} \\
& =\sqrt{\frac{E_{i}}{T}} \sum_{m=0}^{K-1} \alpha_{i m} a_{i m} e^{j(2 \pi / T) m t+\theta_{i m}}, \quad 0<t<T .
\end{aligned}
$$

The received signal after OFDM demodulation and phase compensation can be written in the form [25]

$$
\begin{aligned}
y_{k} & =\frac{1}{\sqrt{T}} \int_{0}^{T} r(t) e^{-j\left((2 \pi / T) k t+\theta_{k}\right)} d t \\
& =\sqrt{E_{c}} \alpha_{k} a_{k}+\mathrm{ICI}+\eta+W \\
& =\sqrt{E_{c}} \alpha_{k} a_{k}+\sum_{i=0}^{I-1} \sqrt{E_{i}} \alpha_{i k} a_{i k} e^{j \psi_{i k}}+W,
\end{aligned}
$$

where $\eta$ is the multiuser interference term, $\psi_{i k}=\theta_{i k}-\theta_{k}$, and $W$ represents the noise term, which has the same statistical characteristic as $W(t)$. Since the channel is static during the symbol interval, we can assume that the ICI of the OFDM is zero. Note that the phases $\theta_{i k}$ and $\theta_{k}$ in (5) and (8) are modeled as random variables uniformly distributed between 0 and $2 \pi$. It is easy to prove that $\psi_{i k}$ is also a random variable uniformly distributed between 0 and $2 \pi$.

To simplify the derivation of the probability of error for the MB-OFDM UWB system, the linearity property of the convolutional codes is used. That is, we assume that the all-zero sequence is transmitted and we determine the probability of error in deciding in favor of another sequence. Note that $a_{k} \in\{ \pm 1, \pm j\}$ and $a_{i k} \in\{ \pm 1, \pm j\}$. The input to the Viterbi decoder can be expressed as

$$
\begin{aligned}
g_{k} & =\operatorname{Re}\left(y_{k}\right)=-\alpha_{k} \sqrt{E_{c}}+\operatorname{Re}(\eta)+\operatorname{Re}(W) \\
& =-\alpha_{k} \sqrt{E_{c}}+\widetilde{\eta}+\widetilde{W} \\
& =-\alpha_{k} \sqrt{E_{c}}+\sum_{i=0}^{I-1} \sqrt{E_{i}} \alpha_{i k}\left[ \pm \cos \psi_{i k} \mp \sin \psi_{i k}\right]+\widetilde{W},
\end{aligned}
$$


where $\widetilde{W}$ is the real white noise, whose double-sided PSD is $N_{w} / 2$. The probability of error in the pairwise comparison of the metrics for path 0 (the all-zero path) and path 1 (a path differs by $d$ bits interval) is

$$
\begin{aligned}
P(d)= & P\left(\sum_{l=1}^{d} g_{l} \geq 0\right) \\
= & P\left(-\sqrt{E_{c}} \sum_{l=1}^{d} \alpha_{l}+\sum_{l=1}^{d} \sum_{i=0}^{I-1} \sqrt{E_{l i}} \alpha_{l i}\left[ \pm \cos \psi_{l i} \mp \sin \psi_{l i}\right]\right. \\
& \left.+\sum_{l=1}^{d} \widetilde{W} \geq 0\right) .
\end{aligned}
$$

In order to simplify the derivation process, we introduce the random variables $X$ and $Y$ defined as

$$
\begin{gathered}
X=\sqrt{E_{c}} \sum_{l=1}^{d} \alpha_{l}, \\
Y=\sum_{l=1}^{d} \sum_{i=0}^{I-1} \sqrt{E_{l i}} \alpha_{l i}\left[ \pm \cos \psi_{l i} \mp \sin \psi_{l i}\right] .
\end{gathered}
$$

Owing to the large bandwidth of the MB-OFDM system and the interleaving used to this system, all $\alpha_{i}$ and $\alpha_{l i}$ could be considered independent from each other over the $d$ bits interval. From the central limit theorem, the random variables $X$ and $Y$ can be approximated as Gaussian random variables with means and variances given by

$$
\begin{gathered}
\mu_{X}=d \sqrt{\frac{E_{c} \Lambda \pi}{2}}, \quad \mu_{Y}=0, \\
\sigma_{X}^{2}=\left(2-\frac{\pi}{2}\right) d E_{c} \Lambda, \quad \sigma_{Y}^{2}=2 d \sum_{i=0}^{I-1} E_{i} \Lambda_{i k} .
\end{gathered}
$$

Hence, the probability of error in (11) can be reduced to

$$
P(d)=P(\tilde{Y}-X \geq 0)=Q\left(\sqrt{\frac{\mu_{X}^{2}}{\left(\sigma_{X}^{2}+\sigma_{\tilde{Y}}^{2}\right)}}\right),
$$

where $\tilde{Y} \sim N\left(0, \sigma_{Y}^{2}+d N_{w} / 2\right)$. This equation gives us an approximation on the expected probability of deciding in favor of a path of Hamming distance $d$ from the transmitted all-zero path. An approximation on the BER of the MBOFDM UWB system then can be calculated as

$$
\begin{aligned}
P_{b} & =\frac{1}{w} \sum_{d=d_{\text {free }}}^{\infty} \lambda_{d} P(d) \\
& =\frac{1}{w} \sum_{d=d_{\text {free }}}^{\infty} \lambda_{d} Q\left(\sqrt{\frac{d \pi}{4[(1-\pi / 4)+1 / \text { SINR } c]}}\right),
\end{aligned}
$$

where $c=w / q$ is the code rate for the MB-OFDM UWB system, $d_{\text {free }}$ is the minimum free distance of the convolutional code used, and $\lambda_{d}$ is the distance spectra of the code. SINR denotes signal to interference plus noise ratio, which is defined as

$$
\operatorname{SINR}=\frac{P_{\text {ave }}}{P_{\text {Int }}+N_{w} / 2}=\frac{E_{b} \Lambda}{\sum_{i=0}^{I-1} E_{i} \Lambda_{i}+N_{w} / 2} .
$$

Note that the BER performance of the MB-OFDM UWB system can be easily computed by substituting the particular SINR into (15). This relatively simple formula could give us good insights into the performance of the MB-OFDM systems in practical channels with multiple interferences.

\section{Simulation and Discussion}

Since the system model in Figure 1 includes multiuser interferers (some other MB-OFDM UWB signals), multipath fading channels, and Viterbi convolution encoder/decoder for various information data rates, the system model is too complicated and time-consuming to perform computer simulation. According to the derivation in Section 2, we know that it is relatively simple for us to evaluate the BER performance of MB-OFDM UWB systems by substituting the particular SINR into (15). Based on this method, we perform some simulation to investigate the effects on the BER performance of MB-OFDM UWB system for the choice of the CCL of the convolutional encoder, the length of the cyclic prefix, and the multiuser environments of two or more interferers.

The parameters of the simulated MB-OFDM systems are summarized in Table 1 [5]. Four Saleh-Valenzuela (S-V) channel models, CM1 CM4, are used in our BER performance simulations. According to the documents provided by the IEEE 802.15.3a group, the parameters of these simulation channels are listed in Table $2[17,18]$. Among these channel models, CM1 model is based on line-of-sight (LOS) measurements and CM2 CM4 are based on non-LOS (NLOS) measurements. By using the simulation parameters listed in Tables 1 and 2, we could easily calculate values of $E_{c}, E_{i}$, and $N_{w} / 2$, that is, the transmitting energy per coded bit, the energy per interference bit, and the double-sided PSD of white Gaussian noise, respectively. The transmitting power of each interferer is assumed to be the same in our simulations.

3.1. The Effect of the CCL of the Convolutional Encoder. In the multiuser environments, since users adopt the different timefrequency code (TFC), the bandwidth of the transmitting signals will not be overlap. We assume the transmitting power and data rate are the same among the multiple users and the transmitted signals are synchronous. The value of the SINR depends on the transmitting power of interference users, the distance between interferer and receiver, and the distance between transmitter and receiver. In general, the package error rate (PER) of the wireless communication systems should be lower than $8 \%$ to comply with FCC regulations. This PER corresponds to the bit error rate (BER) of $8.1424 \times$ $10^{-5}\left(10^{-4.09}\right)$ in the MB-OFDM UWB systems. Figures 2 and 3 show the effects of the different CCL of the convolutional code used in the MB-OFDM UWB systems. From Figure 2, 
TABLE 1: Simulation parameters of the MB-OFDM system.

\begin{tabular}{lcc}
\hline & MB-OFDM group 1 (3-band) & \\
\hline Data transmission rates $\left(R_{b}\right)$ & $110 \mathrm{Mbps}$ & $200 \mathrm{Mbps}$ \\
Average noise power & $-87 \mathrm{dBm}$ & $-84.4 \mathrm{dBm}$ \\
Maximum transmitted power $\left(P_{T}\right)$ & $-10.3 \mathrm{dBm}$ \\
Tx antenna gain $\left(G_{T}\right)$ & $0 \mathrm{dBi}$ \\
Rx antenna gain $\left(G_{R}\right)$ & $0 \mathrm{dBi}$ \\
Central frequency & $3882 \mathrm{MHz}$ \\
Path losses of wireless distance per meter $\left(L_{1}\right)$ & $44.2 \mathrm{~dB}$ \\
Path losses of wireless distance $(d$ meters $)\left(L_{2}\right)$ & $6 \mathrm{~dB}(d=2)$ \\
Average receiving power $\left(P_{R}\right)$ & $12 \mathrm{~dB}(d=4), 20 \mathrm{~dB}(d=10)$ \\
\hline
\end{tabular}

TABLE 2: Parameters of the UWB channel models.

\begin{tabular}{lcccc}
\hline Model parameters & CM1 & CM2 & CM3 & 0.0667 \\
\hline$\Lambda$ [1/nsec] (cluster arrival rate) & 0.0233 & 0.4 & 2.1 & 0.0667 \\
$\lambda[1 /$ nsec $]$ (ray arrival rate) & 2.5 & 0.5 & 14 & 2.1 \\
$\Gamma$ (cluster decay factor) & 7.1 & 5.5 & 7.9 & 24 \\
$\gamma$ (ray decay factor) & 4.3 & 6.7 & 12
\end{tabular}

CM1 model is based on LOS (0-4 m) channel measurement.

CM2 model is based on NLOS $(0-4 \mathrm{~m})$ channel measurement.

CM3 model is based on NLOS (4-10 m) channel measurement.

CM4 model is generated to fit a 25 ns RMS delay spread to an extreme NLOS multipath channel.

we can see that BER conforms to the requirement of the FCC regulations under the condition of CCL $\geqq 3$ and SINR $\geqq 7 \mathrm{~dB}$ for the MB-OFDM UWB system with data rate of $110 \mathrm{Mbit} / \mathrm{s}$. If the data rate of the MB-OFDM UWB system increases up to $480 \mathrm{Mbit} / \mathrm{s}$, the system needs CCL $\geqq 10$ and SINR $\geqq 18 \mathrm{~dB}$ to conform to the BER requirement of the FCC regulations, as shown in Figure 3. The BER of the system would be decreased if the longer length of the CCL is used, due to the longer buffer space of convolutional encoder, at the cost of more complexities of the encoding/decoding circuits of the convolutional code. Therefore, we need carefully to choose the CCL for the MB-OFDM UWB systems.

\subsection{The Received Multipath Energy for Multipath Fading} Channels. Four S-V channel models, CM1 CM4, are used in our BER performance simulations. The more the receiver length is, the more the energy of fading paths is collected at the receiver. For the MB-OFDM UWB systems, the length of cyclic prefix determines the energy level of the paths collected at the receiver. Figure 4 shows the curves of received multipath energy versus the length of the cyclic prefix for four $\mathrm{S}-\mathrm{V}$ channel models. From Figure 4, we can see that if the receiver length is set to $10 \mathrm{nsec}$, the received multipath energy can reach $100 \%, 97 \%, 84 \%$, and $63 \%$ for CM1, CM2, CM3, and CM4, respectively. Figure 5 shows the BER performance for different levels of received multipath energy in the cases of $\mathrm{CCL}=7$ and the data rate of $110 \mathrm{Mbit} / \mathrm{s}$. As shown in Figure 5, the MB-OFDM UWB system with the received multipath energy of $60 \%$ reaches the BER of $10^{-4.09}$ at the SINR = $9 \mathrm{~dB}$. Figure 6 shows the BER performance for different

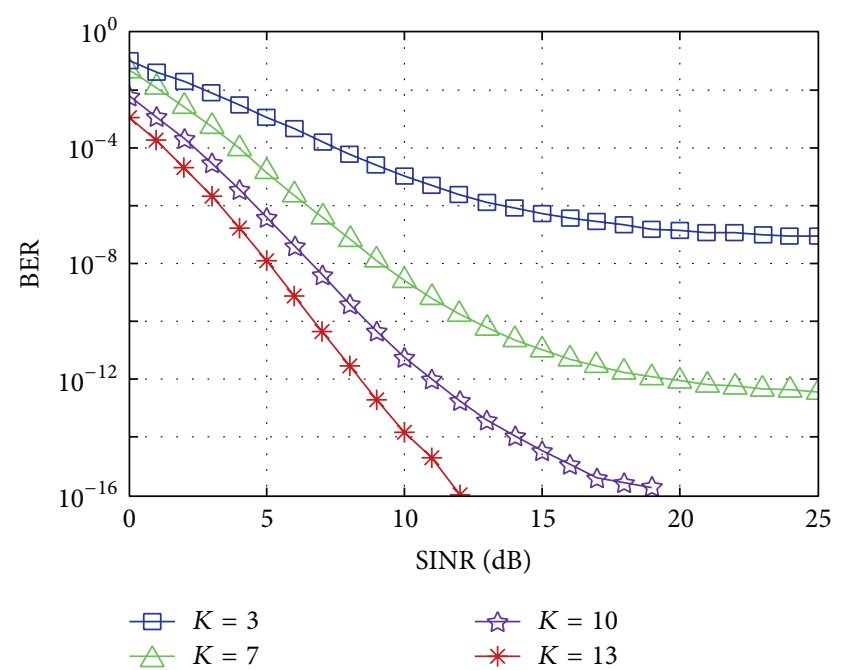

FIGURE 2: BER curves for different CCLs with the data rate of $110 \mathrm{Mbit} / \mathrm{s}$.

levels of received multipath energy in the cases of CCL = 7 and the data rate of $480 \mathrm{Mbit} / \mathrm{s}$. From this figure, we can see that all the curves do not satisfy the BER requirement of the FCC regulations. Therefore, the level of multipath energy collection should be carefully chosen according to the channel conditions of the MB-OFDM UWB system.

3.3. Multiuser Interference Environment: Two Users, One Receiver and One Interferer. Figure 7 shows the relative positions of the multiuser environments for two users. User ${ }_{0}$ is 


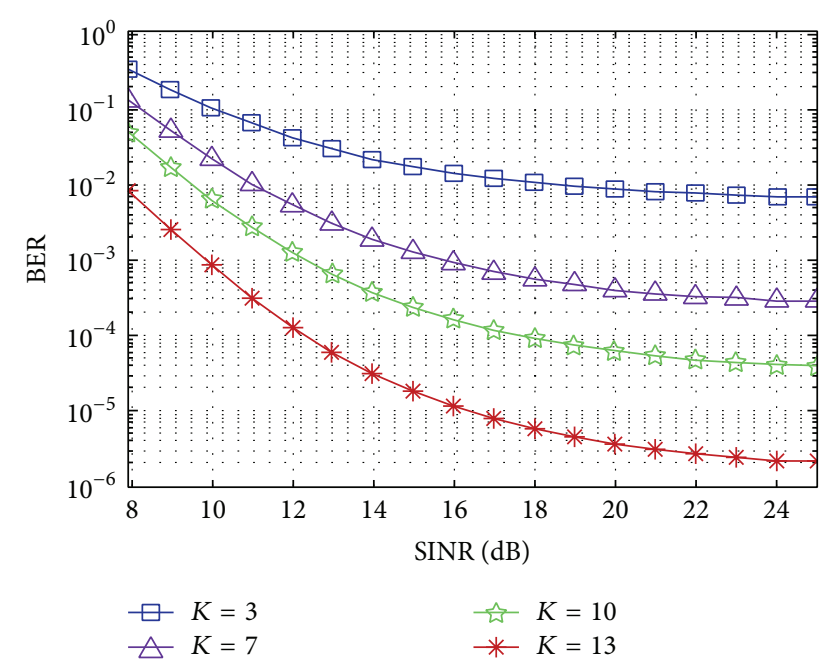

FIgURE 3: BER curves for different CCLs with the data rate of $480 \mathrm{Mbit} / \mathrm{s}$.

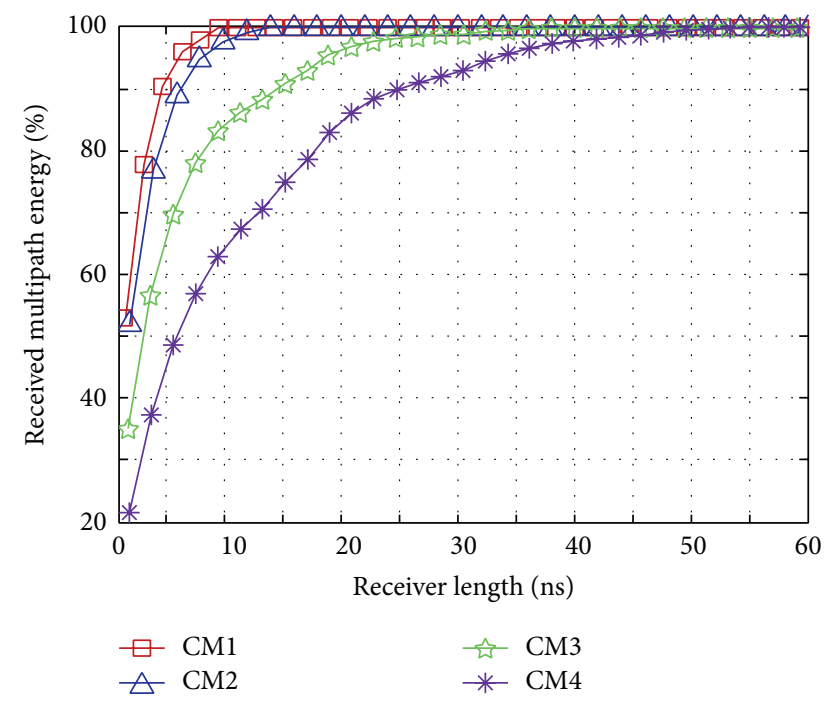

FIGURE 4: Received multipath energies for different UWB channel models.

assumed to be the receiver of interest and user $_{1}$ is interferer. The distance between the user and user $_{1}$ is $d_{1}$. The average received interference power of user $_{0}$ from the interferer decays as a power law of the distance of $d_{1}$. Hence, the equation of SINR could be changed to [25]

$$
\operatorname{SINR}=\frac{E_{c} \Lambda}{\sum_{i=1}^{I} E_{i}\left(d_{i}\right)^{-n} \Lambda_{i}+N_{w} / 2},
$$

where $n$ is the path loss exponent, which typically ranges from 2 to 4 in wireless communication systems. From the channel model used in our analysis, $n$ is assumed to be 1.7 for LOS channels and $n=3.5$ for NLOS channels. The BER performances for different distances of $d_{1}$ and $r$ in the MBOFDM UWB systems with data rates of 110 and $480 \mathrm{Mbit} / \mathrm{s}$ are shown in Figures 8 and 9, respectively. As shown in Figure 8 , we can see that the BER performance is less than

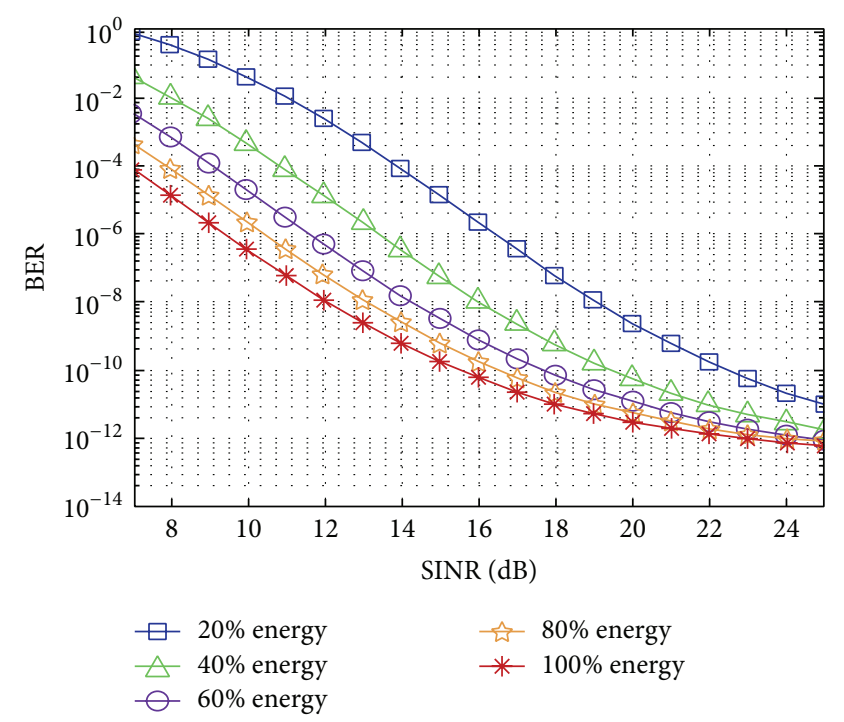

FIGURE 5: BER curves for different received multipath energy with the data rate of $110 \mathrm{Mbit} / \mathrm{s}$ and $\mathrm{CCL}=7$.

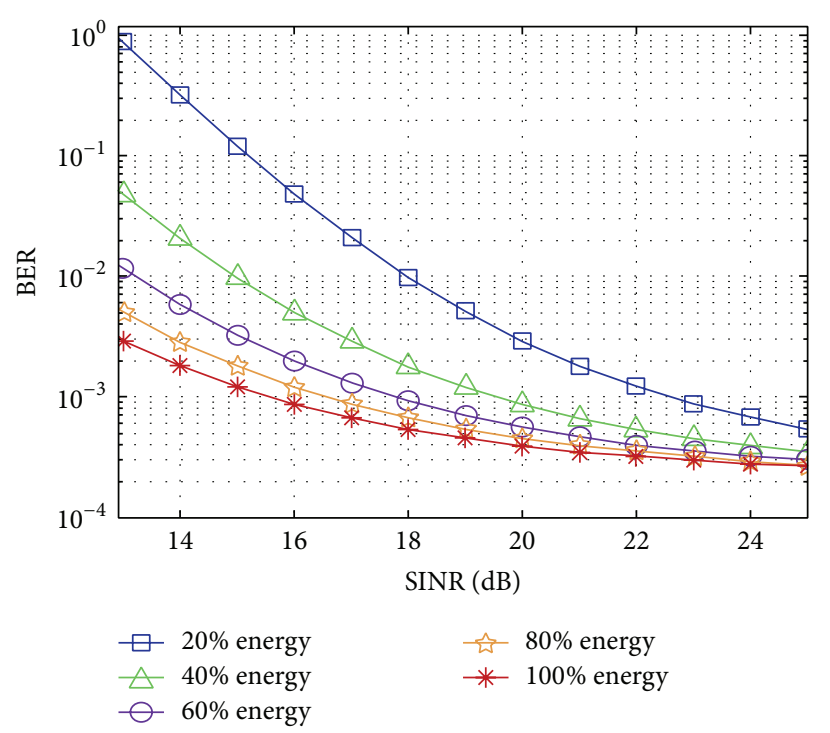

FIGURE 6: BER curves for different received multipath energy with the data rate of $480 \mathrm{Mbit} / \mathrm{s}$ and $\mathrm{CCL}=7$.

$10^{-4.09}$, which is conformed to FCC standards, for $d_{1} \geqq 0.1 \mathrm{~m}$ in the systems with the data rate of $110 \mathrm{Mbit} / \mathrm{s}$ and CCL $=7$. However, as shown in Figure 9, the MB-OFDM UWB systems with data rate of $480 \mathrm{Mbit} / \mathrm{s}$ and $\mathrm{CCL}=7$ have the best BER performance of about $10^{-3}$ for $d_{1}=1 \mathrm{~m}$ and $r=2 \mathrm{~m}$ in NLOS channels. In this situation, the MB-OFDM UWB system would not be able to comply with FCC standards.

3.4. Multiuser Interference Environment: Two Users, One Receiver and Nine Interferers. Figure 10 shows the relative positions of the multiuser environments for ten users. We assume these users are located on the half-circle with the radius of $r$. User 0 is assumed to be the receiver of interest 


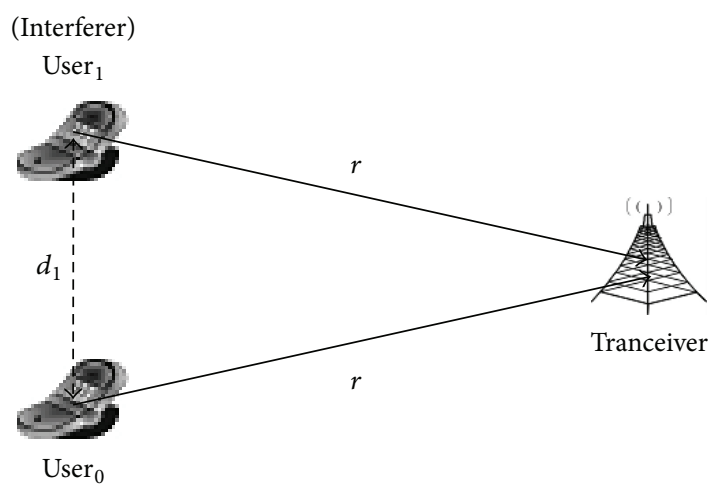

FIgURE 7: The relative positions of the multiuser environments for two users.

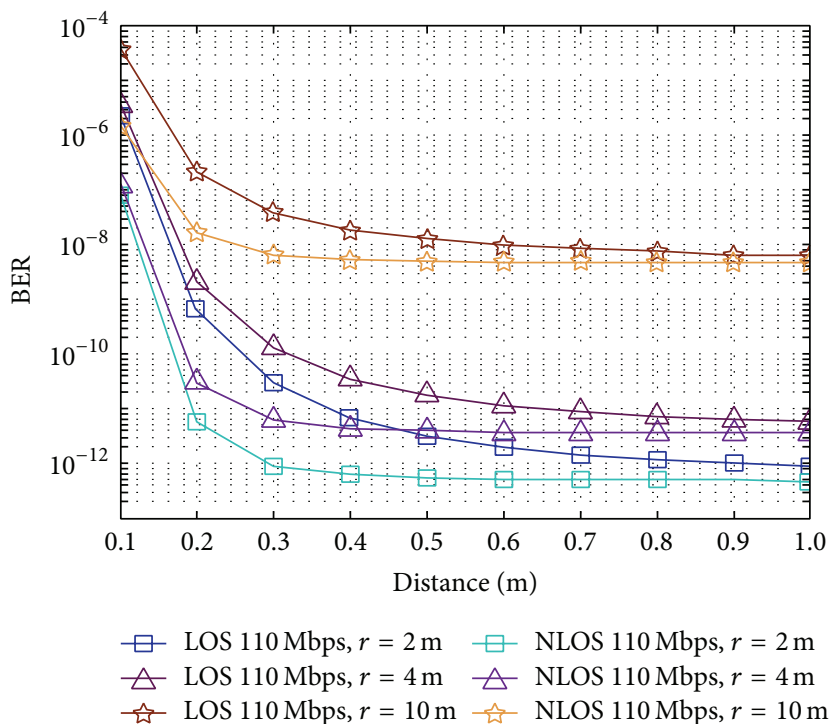

FIGURE 8: BER curves for different transmission distance $r$ with the data rate of $110 \mathrm{Mbit} / \mathrm{s}$ and $\mathrm{CCL}=7$.

and the other users are interferers. The propagation distances between user ${ }_{0}$ and interferers user $_{1}$, user $_{2}, \ldots$, user $_{9}$ are denoted by $d_{1}, d_{2}, \ldots, d_{9}$, respectively. $d_{1}=0.1 \mathrm{~m}$ is set for the simulations of the MB-OFDM UWB system with the data rate of $110 \mathrm{Mbit} / \mathrm{s}$ and $\mathrm{CCL}=7$. Figure 11 shows the BER performance of the MB-OFDM UWB system versus the number of the interference sources for LOS and NLOS channels. The distances of $d_{2}, d_{3}, \ldots, d_{9}$ are increased incrementally with the value of $0.1 \mathrm{~m}$.

From Figure 11, we can see that, for the transmitting distance $r=4 \mathrm{~m}$ and LOS channels, the MB-OFDM UWB system conforms to the BER performance requirement of the FCC standard with a maximum number of interference sources of 5. In NLOS channels, the MB-OFDM UWB system could conform to the FCC BER performance requirement for 9 interference sources and $r=10 \mathrm{~m}$. The BER performance of the MB-OFDM UWB system with data rate of $480 \mathrm{Mbit} / \mathrm{s}$ and $\mathrm{CCL}=7$ is shown in Figure 12. In this simulation, the distance of $d_{1}$ is chosen to be $0.5 \mathrm{~m}$ according to the simulation results

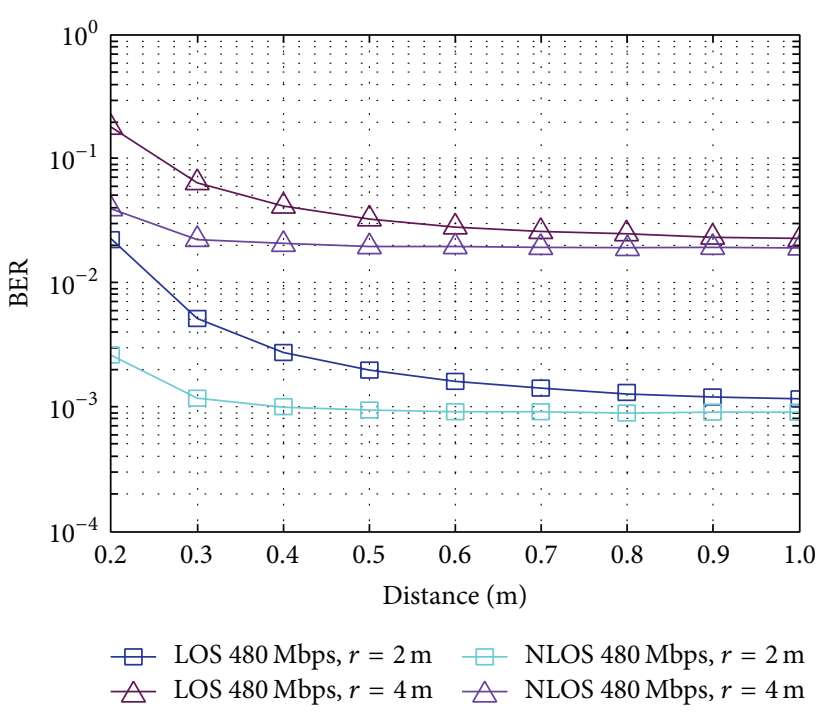

FIGURE 9: BER curves for different transmission distance $r$ with the data rate of $480 \mathrm{Mbit} / \mathrm{s}$ and $\mathrm{CCL}=7$.

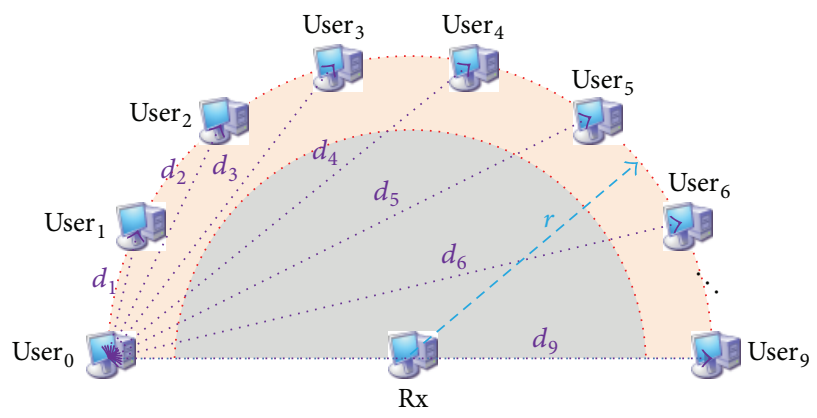

FIGURE 10: The relative positions of the multiuser environments for ten users.

of the two-user case as shown in Figure 9. From this figure, we can see that, for NLOS channels, the BER performance of the MB-OFDM UWB system would be almost the same, as the number of interference sources is increased. However, for LOS channels, the BER performance of the MB-OFDM UWB system would increase, as the number of interference sources is increased. The MB-OFDM UWB systems with the data rate of $480 \mathrm{Mbit} / \mathrm{s}$ and $\mathrm{CCL}=7$ have the best BER performance of about $10^{-3}$ for NLOS channels.

\section{Conclusion}

The formula of the BER performance of the MB-OFDM UWB systems with multiuser interferences is proposed for LOS and NLOS multipath fading channels. We have discussed the effects on the BER performance for the choice of the CCL of the convolutional encoder, the length of the cyclic prefix, and the multiuser environments of two or more interferers. From the simulation results, we can see that the longer CCL would cause better BER performance of the MB-OFDM UWB systems, however, at the cost of more complexities of the encoding/decoding circuits. It also could be noted from 


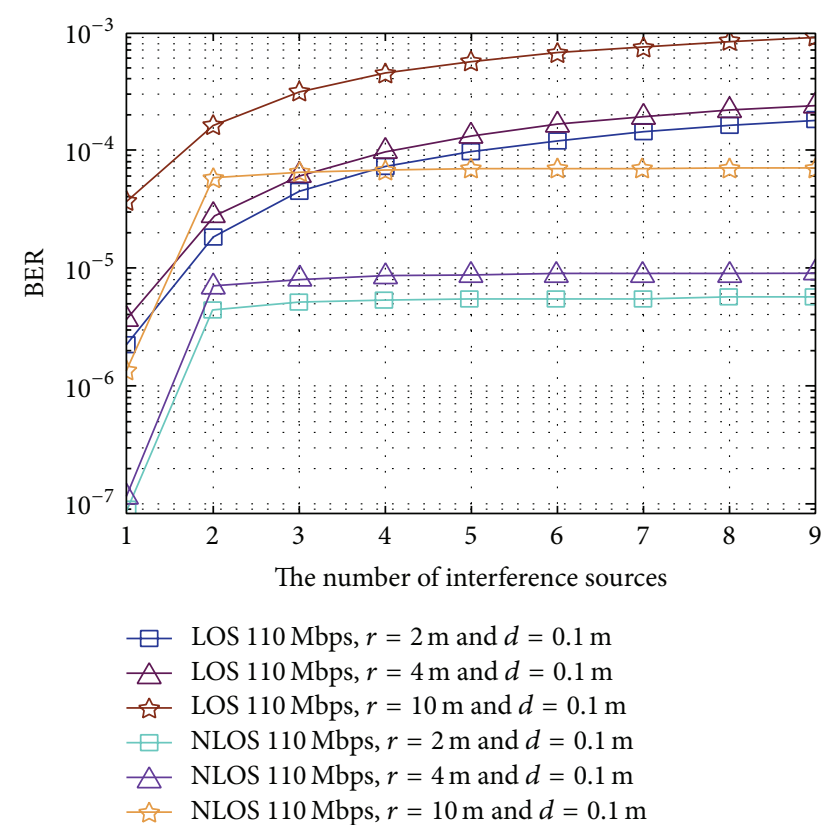

FIGURE 11: BER curves for different $r$ and $d_{1}$ with the data rate of $110 \mathrm{Mbit} / \mathrm{s}$ and CCL $=7$ under LOS/NLOS channels.

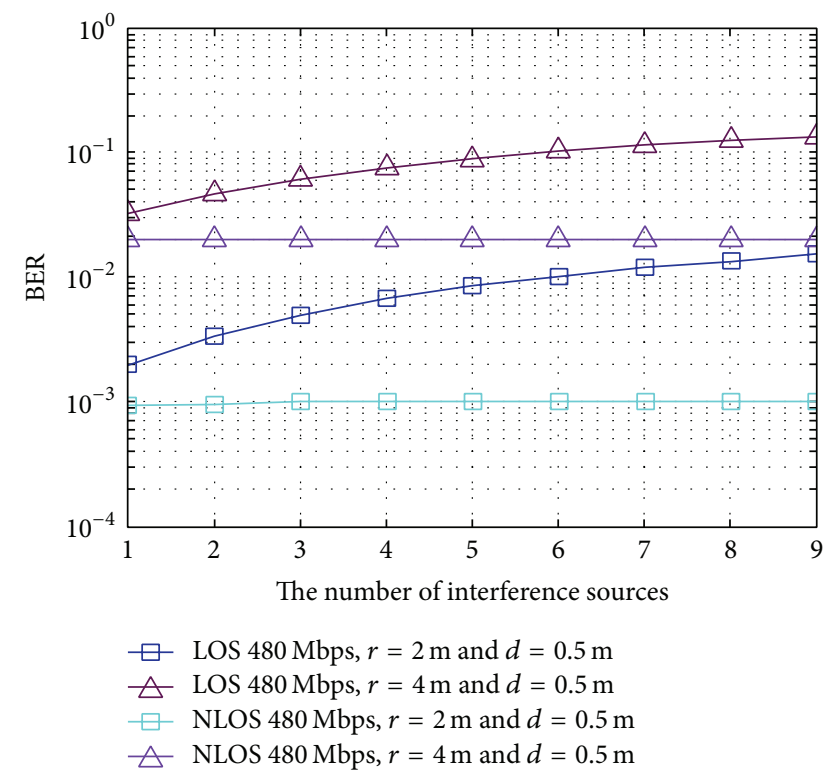

FIGURE 12: BER curves for different $r$ and $d_{1}$ with the data rate of $480 \mathrm{Mbit} / \mathrm{s}$ and CCL $=7$ under LOS/NLOS channels.

these simulation results that the higher data rates would increase the BER of the MB-OFDM UWB system. Four UWB multipath fading channels and the choice of the cyclic prefix have been investigated for the BER performance of the MB-OFDM UWB system. The simulated results demonstrate that we can obtain the BER performance that conforms to the requirements of the FCC standards by appropriately choosing the length of the cyclic prefix of the system. The BER performances for the multiuser environments with LOS and NLOS channels are also investigated in this paper. These simulation results provide us with useful information for choosing the parameters of the MB-OFDM UWB system for multiuser interference and multipath fading channels.

\section{Disclosure}

The authors have no affiliations with or involvement in any organization or entity with any financial interest (such as honoraria; educational grants; participation in speakers' bureaus; membership, employment, consultancies, stock ownership, or other equity interests; and expert testimony or patent-licensing arrangements) or nonfinancial interest (such as personal or professional relationships, affiliations, knowledge, or beliefs) in the subject matter or materials discussed in this paper.

\section{Conflict of Interests}

The authors declare that there is no conflict of interests regarding the publication of this paper.

\section{Authors' Contribution}

All authors participated in the data analysis and/or interpretation. Yuan Ouyang and Chuan-Chih Liu contributed to the conception and design of study. Wen-Piao Lin and ChuanChih Liu contributed to the acquisition of data (laboratory or clinical). Yuan Ouyang and Wen-Piao Lin drafted the paper, made critical revision, and approved the final version of the paper.

\section{Acknowledgments}

The authors would like to acknowledge the support of the Ministry of Science and Technology (MOST) of the Republic of China under Grants NSC 102-2221-E-182-065MY3, NSC 101-2221-E-182-048-MY2, and MOST 103-2221-E182-013. The authors also wish to acknowledge the facilities and financial support from High Speed Intelligent Communication (HSIC) Research Center in Chang Gung University, Taiwan.

\section{References}

[1] High Rate Ultra Wideband PHY and MAC Standard, ECMA Standard 368, 3rd edition, 2008.

[2] K. Siwiak and D. McKeown, Ultra-Wideband Radio Technology, John Wiley \& Sons, New York, NY, USA, 2004.

[3] A. Mehbodniya and S. Aïssa, "Ultra wideband technologies coexistence in Nakagami-m fading channels," IET Communications, vol. 3, no. 7, pp. 1081-1088, 2009.

[4] A. Ghildiyal, B. Godara, K. Amara, R. Dalmolin, and A. Amara, "UWB for low power, short range, in-body medical implants," in Proceedings of the IEEE International Conference on Wireless Information Technology and Systems (ICWITS '10), pp. 1-4, IEEE, Honolulu, Hawaii, USA, September 2010.

[5] P. Gupta and D. K. Mehra, "Kalman filter-based channel estimation and ICI suppression for high-mobility OFDM systems," 
International Journal of Communication Systems, vol. 21, no. 10, pp. 1075-1090, 2008.

[6] E. Saberinia, J. Tang, A. H. Tewfik, and K. K. Parhi, "PulsedOFDM modulation for ultrawideband communications," IEEE Transactions on Vehicular Technology, vol. 58, no. 2, pp. 720-726, 2009.

[7] IEEE802.15 Task Group 3a, "Multiband OFDM physical layer proposal for wireless personal area networks," Doc. no. P802.1503/268r3, 2004.

[8] L. Lampe, A. Nasri, and R. Schober, "Interference from MBOFDM UWB systems: exact, approximate, and asymptotic analysis," in Proceedings of the IEEE International Conference on Ultra-Wideband (ICUWB '06), pp. 261-267, September 2006.

[9] A. Mehbodniya and S. Aïssa, "Effects of MB-OFDM system interference on the performance of DS-UWB," IEEE Transactions on Vehicular Technology, vol. 58, no. 8, pp. 4665-4669, 2009.

[10] H.-Y. Chan, H.-T. Li, C.-W. Chao, and W.-P. Lin, "Bidirectional MB-OFDM ultra-wideband WDM access networks using a time frequency code scheme," in Proceedings of the 6th International Conference on Electrical Engineering/Electronics, Computer, Telecommunications and Information Technology (ECTICON '09), vol. 2, pp. 980-982, Pattaya, Thailand, May 2009.

[11] S. M. R. Islam and K. S. Kwak, "Winner-Hopf interpolation aided kalman filter-based channel estimation for MB-OFDM UWB systems in time varying dispersive fading channel," in Proceedings of the 12th International Conference on Advanced Communication Technology (ICACT '10), vol. 2, pp. 1184-1188, February 2010.

[12] E. Manasseh, S. Ohno, and M. Nakamoto, "Preamble based channel and CFO estimation for MIMO-OFDM systems with null subcarriers," IEICE Transactions on Fundamentals of Electronics, Communications and Computer Sciences, vol. 94, no. 11, pp. 2271-2278, 2011.

[13] D.-H. Kim, K.-M. Kang, and C. Lee, "A multi-band OFDM ultra-wideband SoC in $90 \mathrm{~nm}$ CMOS technology," IEEE Transactions on Consumer Electronics, vol. 57, no. 3, pp. 1064-1070, 2011.

[14] Z. Li, W. Zou, B. Li, Z. Zhou, and X. Huang, "Analysis on coexistence of ultra wideband with OFDM-based communication systems," IEEE Transactions on Electromagnetic Compatibility, vol. 53, no. 3, pp. 823-830, 2011.

[15] S. M. S. Sadough, M. M. Ichir, P. Duhamel, and E. Jaffrot, "Wavelet-based semiblind channel estimation for ultrawideband OFDM systems," IEEE Transactions on Vehicular Technology, vol. 58, no. 3, pp. 1302-1314, 2009.

[16] Z. Wang, Y. Xin, G. Mathew, and X. Wang, "A low-complexity and efficient channel estimator for multiband OFDM-UWB systems," IEEE Transactions on Vehicular Technology, vol. 59, no. 3, pp. 1355-1366, 2010.

[17] J. Foester, "Channel modeling sub-committee report final," IEEE 802.15-02/490r1-SG3, IEEE, 2003.

[18] A. F. Molisch, J. R. Foerster, and M. Pendergrass, "Channel models for ultrawideband personal area networks," IEEE Wireless Communications, vol. 10, no. 6, pp. 14-21, 2003.

[19] A. A. M. Saleh and R. A. Valenzuela, "A statistical model for indoor multipath propagation," IEEE Journal on Selected Areas in Communications, vol. 5, no. 2, pp. 128-137, 1987.

[20] O.-S. Shin, S. S. Ghassemzadeh, L. J. Greenstein, and V. Tarokh, "Performance evaluation of MB-OFDM and DS-UWB systems for wireless personal area networks," in Proceedings of the IEEE
International Conference on Ultra-Wideband (ICU '05), pp. 214219, IEEE, September 2005.

[21] W. P. Siriwongpairat, W. Su, and K. J. R. Liu, "Performance characterization of multiband UWB communication systems using poisson cluster arriving fading paths," IEEE Journal on Selected Areas in Communications, vol. 24, no. 4, pp. 745-751, 2006.

[22] F. C. B. F. Müller, A. Klautau, and C. R. C. M. Da Silva, "Performance analysis of a multiband OFDM UWB system in the presence of narrowband interference," in Proceedings of the IEEE Global Telecommunications Conference (GLOBECOM '09), pp. 1-5, December 2009.

[23] R. Kaneko and F. Maehara, "Theoretical derivation of bit error rate in MB-OFDM UWB system," in Proceedings of the IEEE Wireless Telecommunications Symposium (WTS '09), pp. 1-5, IEEE, Prague, Czech Republic, April 2009.

[24] Y. Li, J. M. Rabaey, and A. Sangiovanni-Vincentelli, "Analysis of interference effects in MB-OFDM UWB systems," in Proceedings of the IEEE Wireless Communications and Networking Conference (WCNC '08), pp. 165-170, April 2008.

[25] G. Zeng, F. A. Cassara, and P. Voltz, "Probability of error for MB-OFDM system in the presence of multi-path fading and multi-user interference," in Proceedings of the 9th International Conference on Advanced Communication Technology (ICACT '07), vol. 3, pp. 2079-2084, Gangwon-Do, Republic of Korea, February 2007. 


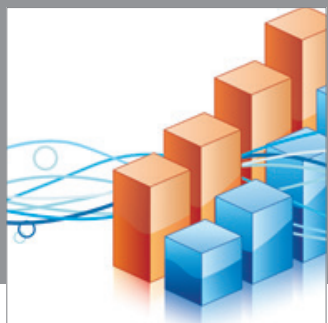

Advances in

Operations Research

mansans

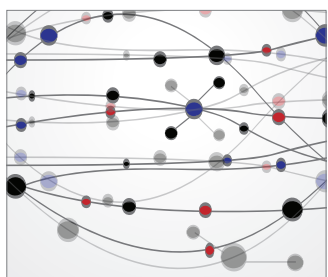

The Scientific World Journal
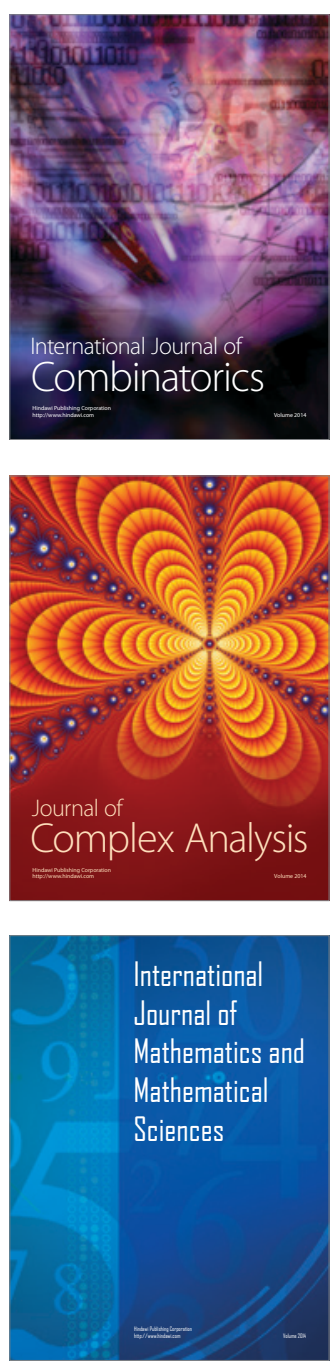
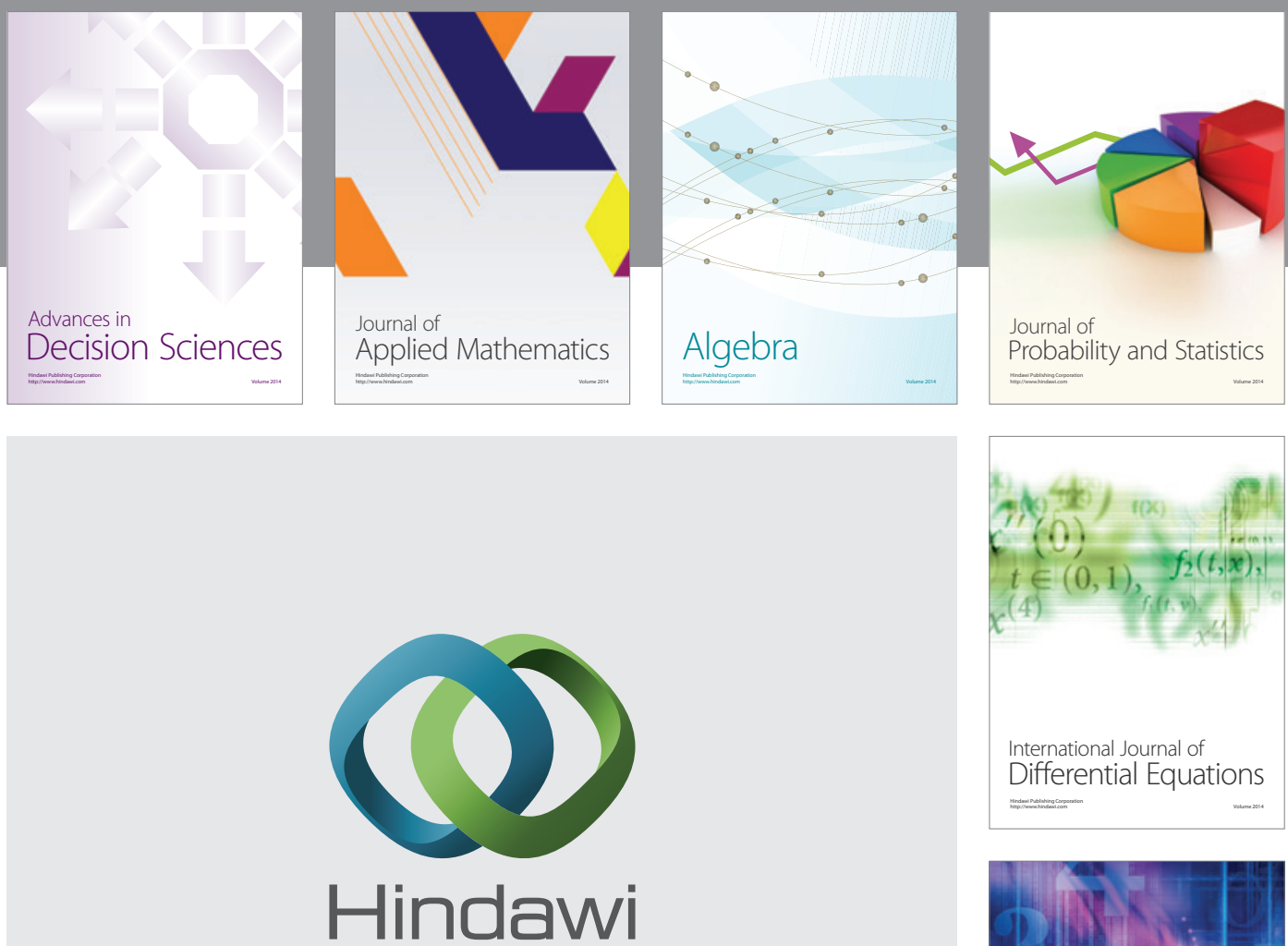

Submit your manuscripts at http://www.hindawi.com
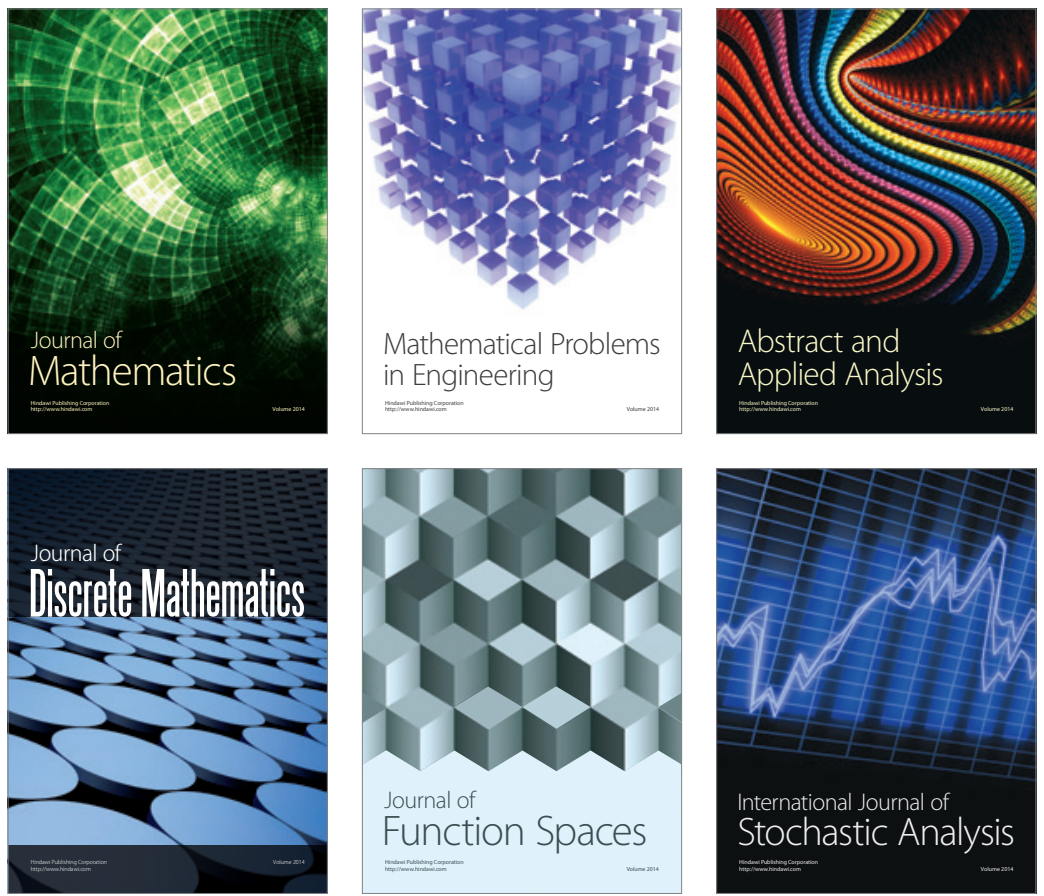

Journal of

Function Spaces

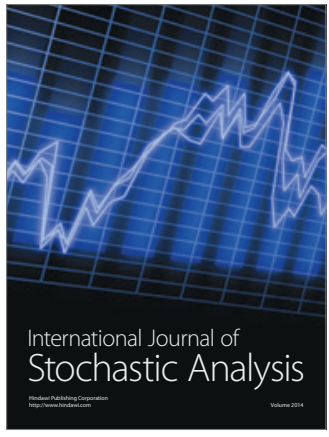

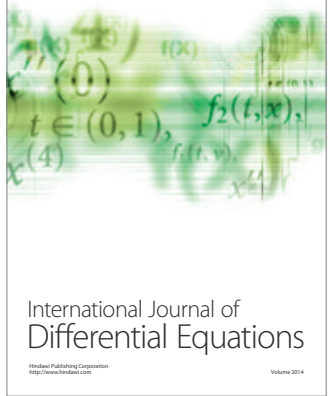
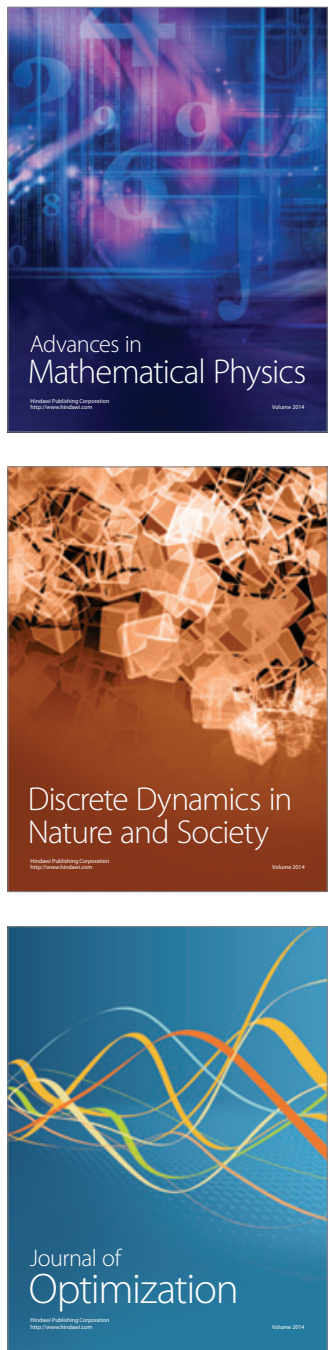